\title{
Characterization of Stevens-Johnson Syndrome and Toxic Epidermal Necrolysis Among Patients Admitted to Kenyatta National Hospital: A Retrospective Cross-Sectional Study
}

\author{
Kenneth Irungu $^{1} \cdot$ David Nyamu $^{2} \cdot$ Sylvia Opanga ${ }^{2}$
}

Published online: 11 April 2017

(C) The Author(s) 2017. This article is an open access publication

\begin{abstract}
Background Stevens-Johnson syndrome (SJS) and toxic epidermal necrolysis (TEN) are severe cutaneous reactions. There is scant literature on the characteristics and causes of these conditions in Kenyatta National Hospital.

Objective The aim of this study was to determine the prevalence, risk factors, and etiologies of SJS/TEN among patients admitted to Kenyatta National Hospital.

Methods A retrospective cross-sectional study was done to find the characteristics and causes of severe cutaneous reactions among patients admitted to Kenyatta National Hospital. Universal sampling was employed, whereby all 115 patients with severe cutaneous reactions between June 2006 and June 2016 were studied. Information collected included participants' sociodemographic variables, clinical characteristics of the disease, and the possible triggers. Data were analyzed using STATA version 13 at $p \leq 0.05$. Results The mean age of patients was 31 years $( \pm 20)$. Low case numbers precluded statistically significant results; however, females represented $59.1 \%$ of patients, and $46.1 \%$ of patients were diagnosed between the ages of 21 and 40 years. SJS occurred in $47 \%$ of patients followed by TEN in $33.9 \%$ and SJS/TEN overlap in $19.1 \%$. Drugs were determined to be the causative agent in $94.8 \%$ of the severe cutaneous reactions followed by infectious agents at $5.2 \%$, principally $\mathrm{HIV}$, herpes simplex virus 1 , and
\end{abstract}

Kenneth Irungu

kariuki.kenneth@gmail.com

1 Ministry of Health, Karatina Sub-County Referral Hospital, P.O. Box 133-10101, Karatina, Kenya

2 Department of Pharmaceutics and Pharmacy Practice, School of Pharmacy, University of Nairobi, P.O. Box 19676-00202, Nairobi, Kenya mycoplasma. The most common drugs implicated were sulfonamides $(26.1 \%)$ and nevirapine $(15.7 \%)$.

Conclusion Numerically, SJS was the most common subtype of SJS/TEN in Kenyatta National Hospital and was usually attributed to use of drugs, especially sulfonamides. Severe cutaneous reactions were observed more frequently in females and in patients aged between 21 and 40 years, indicating that emphasizing surveillance and medication counselling in these patient populations could be beneficial.

\section{Key Points}

Most of the drugs implicated in causing StevensJohnson syndrome (SJS) and toxic epidermal necrolysis (TEN) were low-cost antibiotics commonly prescribed in our setting and drugs for treating HIV, malaria, and TB (infections which are common in our population), thus putting many people at risk of SJS/TEN.

All patients of all ages should be informed of the symptoms of SJS/TEN and closely monitored for these when starting new medications, especially sulfonamide drugs.

\section{Introduction}

Stevens-Johnson syndrome (SJS) and toxic epidermal necrolysis (TEN) are severe bullous skin reactions considered medical emergencies due to their high mortality rate [1]. They are characterized by mucocutaneous tenderness, erythema, hemorrhagic erosions, and necrotic 
epidermal detachment [1]. They are both rare with SJS and TEN having a global incidence of 1-6 and 0.4-1.2 cases per million person years, respectively [2]. Cutaneous drug reactions have a prevalence of $8.259 \%$ and these reactions range from urticaria and erythema to SJS and TEN [3].

The average age groups for TEN and SJS in West Germany have been documented as 63 and 25 years, respectively. Women in this region are at a higher risk for TEN than men at a ratio of $2: 1$, while the converse is true for SJS [4]. In Japan, the average ages for TEN and SJS were observed to be 56.6 and 55.1 years, respectively, with women being more predisposed to SJS than men at a ratio of 1:0.7. There was an equal sex ratio for TEN [5]. In a West African study, the average age group for both SJS and TEN was found to be 32.3 years with a female:male ratio of 1:0.6 [6]. Genetic factors also play a role, with human leukocyte antigens being implicated in SJS and TEN in patients of Asian ancestry. In addition, a slow N-acetylation phenotype predisposes to sulfonamide reactions [7].

Studies have revealed that $80 \%$ of the SJS/TEN cases are caused by drugs, mostly antibacterial sulfonamides, $\beta$ lactams, and also nevirapine [8]. The risk increases when there is co-infection with either mycoplasma pneumonia, herpes simplex virus 1 (HSV-1) or HIV virus, which are also independent etiologies for SJS/TEN [8].

There is scant published local data on the possible causes for SJS and TEN. The main objective of this study was to characterize SJS/TEN and identify their etiologies in Kenyatta National Hospital (KNH).

\section{Methodology}

The study design was a retrospective cross-sectional study in the KNH medical records department. Patients who met the inclusion criteria (children and adults who were diagnosed and treated for SJS/TEN at KNH from June 2006 to June 2016) were included in the study. One hundred and fifteen patients met the criteria. In order to identify these patients, files of SJS/TEN patients were retrieved using the International Classification of Diseases-Adapted Coding Modification 10 (ICDA-10) for L51.1 (SJS) and L51.2 (TEN). Only 89 patient files were obtained using this method. A further search for ICDA-10 skin diagnoses with bullous dermatologic erythematous conditions not otherwise specified (L51.9) yielded 26 more patients. Further review of the excluded files yielded no more SJS/TEN patient files. For a patient to be diagnosed as having SJS they must have had symptoms of acute erythematous mucosal and skin erosions or bullae which are target-like in appearance extending to $<10 \%$ of the body surface area. For SJS/TEN overlap the patient should have had similar lesions covering $10-30 \%$ of the body surface area and for
TEN they should have had extensive epidermal detachment of $>30 \%$ body surface area in addition to the above symptoms. Patient medication histories and a short time lapse from intake of the drug to development of symptoms (2-14 days) confirmed the drugs as causative agents while the history of other illness confirmed, without intake of anti-retrovirals, HIV as the etiology. Furthermore, a Naranjo algorithm was used to determine causality of adverse drug reaction as had been indicated in the file [9]. Mycoplasma pneumonia was determined through laboratory tests and HSV-1 was determined through physical examination by a dermatologist.

Pre-designed data collection forms were used to abstract participants' socio-demographics, disease characteristics, subtype of the disease, and causative drugs. Twenty of the SJS/TEN patients' medical files were randomly picked and data abstracted by an independent reviewer on separate data collection forms for comparison with our investigators' forms. Discrepancies were sorted out by consensus. A database was created using MS Excel version 2007 software. This was exported to STATA software version 13 for analysis.

Prevalence of the subtypes SJS, TEN, and SJS/TEN overlap was determined. The mean and median age of the disease at diagnosis were computed. Percentages were calculated for all of the patient characteristics and the causative agents. Inferential data analysis was done using the Chi-square test to compare for differences across causative agents and patient characteristics using STATA software version 13 (Statacorp, 4905 Lakeway Drive College Station, Texas 77845 USA: licensed to Major Nyamai University of Nairobi). The level of significance was set at 0.05 and $p$ values $\leq 0.05$ were considered statistically significant.

\section{Results}

Of the 115 patients, the majority (68) was female. The mean and median ages were $31 \quad( \pm 20)$ and 32 (12-42) years, respectively. More patients were aged 21-40 years (53) than younger or older (33 and 29, respectively) (Table 1 ).

\subsection{Relative Frequency of Various Disease Subtypes Across the Study Population}

While insufficient cases were recorded to reach statistical significance, SJS was observed most frequently, occurring in 54 patients followed by TEN $(n=39)$ and SJS/TEN $(n=22)$. Among the total number of patients with severe cutaneous reactions, only 24 had a severity-of-illness score (SCORTEN) calculated, as shown in Table 2. 
Table 1 Socio-demographic characteristics of the study participants

\begin{tabular}{|c|c|}
\hline Characteristic & $n(\%)$ \\
\hline \multicolumn{2}{|l|}{ Age category } \\
\hline Mean age, years (SD) & $31( \pm 20)$ \\
\hline Median age, years (range) & $32(12-42)$ \\
\hline $21-40$ years & $53(46.1)$ \\
\hline $41-60$ years & $22(19.1)$ \\
\hline$>60$ years & $7(6.1)$ \\
\hline \multicolumn{2}{|l|}{ Sex } \\
\hline Male & 47 (40.9) \\
\hline Female & $68(59.1)$ \\
\hline \multicolumn{2}{|l|}{ Occupation } \\
\hline Salaried & $24(21.1)$ \\
\hline Self-employed & $35(30.7)$ \\
\hline Unemployed & $55(48.3)$ \\
\hline \multicolumn{2}{|l|}{ Education level } \\
\hline Non-formal & $25(23.2)$ \\
\hline Primary & $43(39.8)$ \\
\hline Secondary & $30(27.8)$ \\
\hline College/university & $10(9.3)$ \\
\hline \multicolumn{2}{|l|}{ Year of diagnosis } \\
\hline 2006-2009 & $17(14.9)$ \\
\hline 2010-2013 & $42(36.5)$ \\
\hline 2014-2016 & $56(48.7)$ \\
\hline \multicolumn{2}{|l|}{ Residence } \\
\hline Nairobi & $27(23.5)$ \\
\hline Central & $24(20.9)$ \\
\hline Eastern & $21(18.3)$ \\
\hline Nyanza & $14(12.2)$ \\
\hline Rift Valley & $13(11.3)$ \\
\hline Western & $12(10.4)$ \\
\hline North Eastern & $3(2.6)$ \\
\hline Coast & $1(0.9)$ \\
\hline
\end{tabular}

In keeping with total cases in 21- to 40-year-olds being more frequent than in other age groups, there were greater numbers of cases of each subtype within this age group than in other age brackets (see Table 4). Numerically, more cases of SJS and TEN occurred in females and more cases of SJS/TEN overlap occurred in males (Table 4).

\subsection{Relative Frequency of Exposure to Agents Known to Cause SJS/TEN in the Study Population}

One hundred and nine $(94.8 \%)$ of the SJS/TEN cases were thought to be caused by use of drugs. Twelve (10.4\%) of the patients in the study had SJS/TEN attributable to intake of more than one drug. The remaining six cases of SJS/
Table 2 Disease characteristics of the study population

\begin{tabular}{lc}
\hline Characteristic & $n(\%)$ \\
\hline Subclass of the disease $(n=115)$ & $54(47.0)$ \\
SJS & $22(19.1)$ \\
SJS/TEN overlap & $39(33.9)$ \\
TEN & \\
SCORTEN & $5(20.8)$ \\
1 & $11(45.8)$ \\
2 & $5(20.8)$ \\
3 & $2(8.3)$ \\
4 & $1(4.2)$ \\
6 & $2(1.7)$ \\
Method of diagnosis $(n=115)$ & $113(98.3)$ \\
Histopathological & $105(91.3)$ \\
Clinical & $10(8.7)$ \\
Origin of the skin reaction $(n=115)$ & $2(1.7)$ \\
Home & $113(98.3)$ \\
Hospital & 4 days (range $2-14)$ \\
Family history $(n=115)$ & \\
Positive & \\
Negative & \\
Median latency time from exposure to drug to & \\
onset of symptoms & \\
\hline SJS Stevens-Johnson syndrome, TEN toxic epidermal necrolysis \\
\end{tabular}

TEN were thought to be caused by infectious agents, namely HIV $(n=4)$, HSV1 $(n=1)$, and mycoplasma pneumonia $(n=1)$.

As seen in Table 3, use of sulfonamides accounted for the highest number of cases at $25(22.9 \%)$, followed by nevirapine at $18(16.5 \%)$, ciprofloxacin at $13(11.9 \%)$, and penicillins at $8(7.3 \%)$. Other drugs implicated included metronidazole $(n=3)$, lamotrigine $(n=1)$, phenobarbitone $(n=1)$, efavirenz $(n=2)$, meloxicam $(n=1)$, hydrochlorothiazide $(n=1)$, paracetamol $(n=2)$, allopurinol ( $n=1)$, rifampicin/isoniazid/pyrazinamide/ethambutol $(n=3)$, quinine $(n=2)$, magnesium sulphate $(n=1)$, griseofulvin $(n=1)$, streptomycin $(n=1)$, herbal medication $(n=2)$,oral polio vaccine $(n=1)$, and unknown medication $(n=11)$.

\subsection{Association Between Demographic Characteristics and Development of Various Disease Subtypes}

Table 4 compares the demographic characteristics and development of disease subtypes. There were no statistically significant associations between patient's age, gender, and education level on the development of various disease subclasses. 
Table 3 Probable causative agent of SJS/TEN in Kenyatta Hospital patients where a drug was suspected $(N=109)$

\begin{tabular}{lc}
\hline Drug & $\begin{array}{l}\text { SJS/TEN cases attributable to drug, } \\
n(\%)\end{array}$ \\
\hline $\begin{array}{l}\text { Sulfamethoxazole/ } \\
\text { trimethoprim }\end{array}$ & $25(22.9)$ \\
Nevirapine & $18(16.5)$ \\
Ciprofloxacin & $13(11.9)$ \\
Penicillins & $8(7.3)$ \\
Cephalosporins & $6(5.5)$ \\
Artemether/lumefantrine & $6(5.5)$ \\
Sulfadoxine/pyrimethamine & $5(4.6)$ \\
Carbamazepine & $5(4.6)$ \\
Others & $23(21.1)$ \\
Total & $109(100)$ \\
\hline
\end{tabular}

SJS Stevens-Johnson syndrome, TEN toxic epidermal necrolysis

\subsection{Association Between the Prevalence of Various Drugs Implicated in Causation of Disorders and Development of Disease Subtypes}

Table 5 shows the association between the prevalence of various drugs implicated in causation of disorders and development of disease subtypes. Notably, most of the drugs implicated in causing SJS/TEN disease subtypes were antibiotics and drugs used in the treatment of HIV and malaria.

\section{Discussion}

There were a total of 115 patients treated for severe cutaneous reactions over a period of 10 years, representing a prevalence of 11 cases per year. This result mirrors the scarcity of this serious toxidermia observed in other studies $[1,2,4]$. While cases were too infrequent for comparisons to reach statistical significance, $59.1 \%$ of cases were in women, while $40.9 \%$ were in men. This female predominance is similar to findings from other studies done by Saka et al. [6] and Chan [10]. Numerically, there were more females than males with SJS and TEN disease subtypes at 30.4 versus $16.5 \%$ and 20.0 versus $13.9 \%$, respectively. This trend has been seen in another study, where more females had SJS and TEN disease subtypes at 28.2 versus $16.2 \%$ and 29.1 versus $26.5 \%$, respectively [11]. There were, however, no statistically significant associations between patients' sex on the development of various disease subclasses.

The largest number of patients was those aged between 21 and 40 years (46.1\%), compared with $0-20$ years (28.7\%), 41-60 years $(19.1 \%)$, or $>60$ years $(6.1 \%)$. There were also more patients in this age category across the various disease subtypes, a finding that was replicated in the study by Saka et al. [6]. This does not match all-cause age distribution for patients at the hospital, so could possibly be attributed to frequent self-prescribing of medicine in this age group. Our results on the most prevalent age
Table 4 Relationship between demographics and SJS/TEN disease subtype

\begin{tabular}{|c|c|c|c|c|}
\hline \multirow[t]{2}{*}{ Demographic characteristic } & \multicolumn{3}{|c|}{ Disease subclass } & \multirow[t]{2}{*}{$p$ value } \\
\hline & $\begin{array}{l}\text { SJS } \\
n(\%)\end{array}$ & $\begin{array}{l}\text { SJS/TEN } \\
n(\%)\end{array}$ & $\begin{array}{l}\text { TEN } \\
n(\%)\end{array}$ & \\
\hline \multicolumn{5}{|l|}{ Age category (years) } \\
\hline $0-20$ & $15(13)$ & $6(5.2)$ & $12(10.4)$ & \multirow{4}{*}{0.547} \\
\hline $21-40$ & $29(25.2)$ & $8(7)$ & $16(13.9)$ & \\
\hline $41-60$ & $8(7)$ & $5(4.3)$ & $9(7.8)$ & \\
\hline$>60$ & $2(1.7)$ & $3(2.6)$ & $2(1.7)$ & \\
\hline \multicolumn{5}{|l|}{ Sex } \\
\hline Male & $19(16.5)$ & $12(10.4)$ & $16(13.9)$ & \multirow[b]{2}{*}{0.313} \\
\hline Female & $35(30.4)$ & $10(8.7)$ & $23(20)$ & \\
\hline \multicolumn{5}{|l|}{ Occupation } \\
\hline Salaried & $8(7.0)$ & $6(5.2)$ & $10(8.7)$ & \multirow{3}{*}{0.634} \\
\hline Self-employed & $18(15.7)$ & $6(5.2)$ & $11(9.6)$ & \\
\hline Unemployed & $28(24.3)$ & $9(7.8)$ & $18(15.7)$ & \\
\hline \multicolumn{5}{|l|}{ Education level } \\
\hline Non-formal & $11(9.6)$ & $4(3.5)$ & $10(8.7)$ & \multirow{4}{*}{0.154} \\
\hline Primary & $23(20)$ & $6(5.2)$ & $14(12.2)$ & \\
\hline Secondary & $11(9.6)$ & $8(7)$ & $11(9.6)$ & \\
\hline College/university & $7(6.1)$ & $3(2.6)$ & $0(0)$ & \\
\hline
\end{tabular}

SJS Stevens-Johnson syndrome, TEN toxic epidermal necrolysis 
Table 5 Relationship between frequency of drugs being implicated in SJS/TEN causation and disease subtypes

\begin{tabular}{lllll}
\hline Drug & $\begin{array}{l}\text { SJS } \\
n(\%)\end{array}$ & $\begin{array}{l}\text { SJS/TEN overlap } \\
n(\%)\end{array}$ & $\begin{array}{l}\text { TEN } \\
n(\%)\end{array}$ & $p$ value \\
\hline Sulfonamides & $16(13.9)$ & $7(6.1)$ & $15(13)$ & 0.657 \\
Nevirapine & $10(8.7)$ & $4(3.5)$ & $4(3.5)$ & 0.496 \\
Ciprofloxacin & $4(3.5)$ & $2(1.7)$ & $7(6.1)$ & 0.311 \\
Unknown drug $^{\text {B-Lactam antibiotics }}{ }^{\mathrm{a}}$ & $6(5.2)$ & $1(0.9)$ & $4(3.5)$ & 0.839 \\
Artemether/lumefantrine $_{\text {Carbamazepine }}^{7(6.1)}$ & $3(2.6)$ & $4(3.5)$ & 0.869 \\
\hline
\end{tabular}

SJS Stevens-Johnson syndrome, TEN toxic epidermal necrolysis

a $\beta$-Lactam antibiotics-penicillins and cephalosporins group conflict with other studies that show most patients being elderly and attributing this to reduced drug clearance, or to most patients being children because of poor immune status. For instance, one study [10] found more SJS/TEN patients in the $<5$-years-old category, attributing it to viral causes that are more prevalent in this age group, while some findings in West Germany found more TEN in patients aged above 63 years [4].

Nearly half of the patients were seen in the last 2 years because of improved referral systems from different health facilities and reception to our study site. These were put in place by the public health administration that facilitated more patients reaching our study site. Kenyatta National Hospital is the largest referral center in East Africa. The site was appropriate because it is the largest public hospital that provides specialized medical management and treatment services for all conditions and for all ages, both children and adults. It is also the facility with the top medical experts in the country, including dermatologists who are involved in the management of SJS/TEN, hence justifying the referrals to the hospital. SJS/TEN patients from all over the country are also referred here because management of this condition can be appropriately carried out in either the critical care unit or the burns unit if necessary.

Nairobi and Central provinces contributed the highest percentage of patients at 23.5 and $20.9 \%$, respectively. This could be attributable to the proximity of the provinces to the study site and as such most patients were referred for further management owing to the life-threatening nature of the toxidermia.

More patients were also less educated (63.0\%), and a high percentage were unemployed $(48.3 \%)$.These are factors that affect proper medication use and good access to safe efficacious medications [12].

The causative agents of SJS/TEN were determined by checking the original patient medical files since it was a retrospective study. These were considered accurate due to the following reasons: the drugs implicated in our study were consistent with those in other studies. For other drugs like anti-tuberculosis drugs and anti-malarials there is literature implicating them to cause SJS/TEN [6]. There was a reasonable time lapse between exposure to the drugs and development of symptoms. All our patients during their stay at the hospital had been reviewed by teams of dermatologists and medical experts whose acumen we thought was adequate to have determined the causative agents. Also, during data collection, the medical files had been reviewed by two independent reviewers to ascertain that information obtained from the files was accurate.

Our study revealed the probable causes of SJS/TEN to be mostly drugs, accounting for over $90 \%$. Twelve of our patients had SJS/TEN attributable to use of more than one drug. For each of these drugs, a probability of causality of 0.5 was assigned and the latency period for onset of symptoms was determined from intake of the first drug. The median latency for development of symptoms after exposure to the drugs was 4 days with a range of 2-14 days, matching other studies [7, 13]. This is consistent with the latency period for type IV hypersensitivity reaction mediated by $\mathrm{T}$ lymphocytes, the reaction responsible for causing SJS/TEN [2, 7]. The high prevalence of probable drug causes correlates with studies by Roujeau et al. [1], Haddad et al. [14], and Levi et al. [15]. HIV as a cause accounted for $3.5 \%$ of the cases and in a regional study by Saka et al., an association was found between HIV and SJS/TEN [6]. HSV-1 and mycoplasma pneumonia were also confirmed to be associated with SJS/TEN as in other studies [16, 17]. Of particular importance is the fact that the drugs mostly implicated to cause SJS/TEN in our study are antibiotics, anti-retrovirals, anti-tuberculosis drugs, and anti-malarials. All of these are commonly used locally, due to the high prevalence of relevant infectious diseases in this region, suggesting that many people are at risk of SJS/TEN because of using these drugs. While antiretrovirals and anti-tuberculosis drugs are used more frequently in patients aged $21-40$ years, antibiotics and antimalarials are more frequently used in patients aged 0-20 years $[18,19]$. As such, the region's unique 
prescribing patterns likely do not account for the prevalence of SJS/TEN in patients aged 21-40 years as opposed to different age groups, as observed in some other studies $[4,10]$.

Most of the severe cutaneous reactions were attributed to sulfonamides (27.5\%) followed by ciprofloxacin (11.9\%), penicillins (7.3\%), and cephalosporins. The multicentric European study by Roujeau et al. found a significant association between SJS/TEN and antibacterial sulfonamides, aminopenicillins, and quinolones [1]. Nevirapine use was also implicated at $16.5 \%$, as was found by Mockenhaupt et al. in the EuroScar study [20].

Roujeau et al. implicated anticonvulsant medications carbamazepine, phenobarbitone, and phenytoin to cause SJS/TEN, but this did not match our study [1].This could have been due to a lower prevalence of convulsive disorders in our population or differences in study methodologies. This could also be because of genetic differences between our population and the Western and Asian countries. Mockenhaupt et al. had also found significant association between SJS/TEN and lamotrigine, whereas we found only one case linked to its use in our study [20]. This could be attributed to the infrequent use of this drug among the local population since it is not in our treatment guidelines as first-line management of seizures [21].

Anti-tuberculosis medications and antimalarial drugs were notably missing as probable causes of SJS/TEN in the studies done in Europe and Asia, but have featured in our study and also in a multicentric study done by Saka et al. in West Africa [6]. This could be explained by the high prevalence of tuberculosis and malaria in Africa as compared with the other two continents.

Herbal medications sought from traditional healers may have unknown ingredients owing to adulteration and undocumented phytochemical extracts. These herbal medications have been shown to cause SJS/TEN [6] and in our study they were implicated in two cases. This was minimal considering the rampant use of herbal medicines locally. We attributed this to under-reporting of their use by patients to clinicians.

There is a paucity of published data on associations between metronidazole and SJS/TEN. However, in our study it accounted for two cases. This prevalence was low because local anecdotal information on the association between metronidazole and SJS/TEN is high and may have alerted clinicians on its use. Other drugs implicated in our study and not in previous research included magnesium sulfate and griseofulvin. However, it was observed that the patient who reacted to magnesium sulfate had been receiving penicillins earlier, which might have been a confounder. It was also observed that the patient who reacted to griseofulvin had been taking sulfonamides, a possible confounder.
There was a high number of SJS/TEN cases $(n=11)$ caused by unidentified drugs. It was observed that these had been prescribed from far-flung hospitals and no records were available to identify them.

\subsection{Study Limitations}

Information bias arose since the cases were already classified as SJS or TEN according to the medical records. We relied on reported data and the verification was difficult because some of these patients may have suffered from other skin illnesses. This bias was minimized by using a standardized data collection tool that indicated the symptoms required for diagnosis of SJS/TEN. Additionally, twenty random medical files were selected, reviewed, and data extracted to a separate data collection tool by an independent dermatology resident who was not affiliated to the study. Comparisons were made for the two data sets and differences sorted out by consensus. There was also a small sample size which we tried to increase by going back in time over 10 years.

\section{Conclusion and Recommendation}

SJS/TEN are rare severe cutaneous reactions and the most frequently observed disease subtype in our study was SJS. Women and persons aged 21-40 years were the most affected groups within our study population, and drugs, especially sulfur-based medications, were deemed to be the causative agent in over $90 \%$ of the cases. The findings suggest that clinicians should be aware of the side effects of sulfur-based drugs for effective pharmacovigilance among patients who have these medicines prescribed for them. Due to the severity of SJS/TEN, all patients of all age groups taking new medications should undergo close drug monitoring and, based on our study results, this is especially important for women aged 21-40 years. Further studies are warranted in greater sample sizes across Kenya, comparing patient characteristics with the general population, in order to reach statistical significance and to determine any other predisposing factors to SJS/TEN.

Acknowledgements We thank the following for their support: Dr Peter Karimi, Dr Eric Guantai, Samantha Irungu, and the Kenyatta National Hospital medical records staff.

Author's Contributions All authors were involved in drafting the protocol and the interpretation of results. Data were extracted from patient files by KI. Data analysis was carried out by KI with the help of DG and a biostatistician. All authors were involved in the drafting and approval of the final review, with KI being the coordinating author. All authors read and approved the final manuscript. 


\section{Compliance with Ethical Standards}

Funding for the study This study was funded by the authors.

Conflict of interest Kenneth Irungu declares no conflict of interest, David Nyamu declares no conflict of interest, Sylvia Opanga declares no conflict of interest.

Ethical clearance Ethical approval was sought from the Kenyatta National Hospital/University of Nairobi Ethics and Research committee (KNH/UON-ERC) and approval (Ref: KNH-ERC/A/119) was granted on 4th April 2016.

Open Access This article is distributed under the terms of the Creative Commons Attribution-NonCommercial 4.0 International License (http://creativecommons.org/licenses/by-nc/4.0/), which permits any noncommercial use, distribution, and reproduction in any medium, provided you give appropriate credit to the original author(s) and the source, provide a link to the Creative Commons license, and indicate if changes were made.

\section{References}

1. Roujeau J, Kelly J, Naldi L, Rzany B, Stern R, Anderson T, et al. Medication use and the risk of Stevens-Johnson syndrome or toxic epidermal necrolysis. N Engl J Med. 1995;333:1600-7.

2. Harr T, French L. Toxic epidermal necrolysis and StevensJohnson syndrome. Orphanet J Rare Dis. 2010;5:39.

3. Patel T, Thakkah S, Sharmar D. Cutaneous adverse drug reactions in Indian population: a systematic review. Indian Dermatol Online J. 2014;5:76-86.

4. Schopf E, Stuhmer A, Rzany B, Victor N, Zentgraf R, Kapp J. Toxic epidermal necrolysis and Stevens-Johnson syndrome: an epidemiologic study from West Germany. Arch Dermatol. 1991;127:839-42.

5. Yamane Y, Matsukura S, Watanabe Y, Yamaguchi Y. Retrospective analysis of Stevens Johnson syndrome and toxic epidermal necrolysis in 87 Japanese patients: treatment and outcome. Allergol Int. 2016;65:74-81.

6. Saka B, Barro-traore F, Atadokpe FA, Niamba PA, Ade H, Pitche VP, et al. Tropical medicine rounds Stevens-Johnson syndrome and toxic epidermal necrolysis in sub-Saharan Africa: a multicentric study in four countries. Int J Dermatol. 2013;52:575-9.

7. Clinard V. Drug-induced skin disorders. In: US Pharmacist. 2012. https://www.uspharmacist.com/article/drug-induced-skin-disorders. Accessed 10 Dec 2015.
8. Roujeau J, Stern R. Severe adverse cutaneous reactions to drugs. N Engl J Med. 1994;331:1272-85.

9. Naranjo C, Busto U, Sellers E, Sandor P, Ruiz I, Roberts E, et al. A method for estimating the probability of adverse drug reactions. Clin Pharmacol Ther. 1981;30:239-45.

10. Chan H-L. The incidence of erythema multiforme, StevensJohnson syndrome, and toxic epidermal necrolysis. Arch Dermatol Am Med Assoc. 1990;126:43.

11. Yamane Y, Aihara M, Ikezawa Z. Analysis of Stevens-Johnson syndrome and toxic epidermal necrolysis in Japan from 2000 to 2006. Allergol Int. 2007;56:419-25.

12. Kizito M. Assessment of the patient factors impacting on oral anticoagulation therapy among adult outpatients at Kenyatta national hospital. [MPharm Thesis]. University of Nairobi, 2015.

13. Bastuji-garin S, Rzany B, Stern RS, Shear NH, Naldi L, Roujeau J. Clinical classification of cases of toxic epidermal necrolysis, Stevens-Johnson syndrome, and erythema multiforme. Arch Dermatol. 1993;129:92-6.

14. Haddad C, Sidoroff A, Kardaun SH, Mockenhaupt M, Creamer D, Dunant A, et al. Stevens-Johnson syndrome/toxic epidermal necrolysis: are drug dictionaries correctly informing physicians regarding the risk? Drug Saf. 2013;36:681-6.

15. Levi N, Bastuji-Garin S, Mockenhaupt M, Roujeau J-C, Flahault A, Kelly JP, et al. Medications as risk factors of Stevens-Johnson syndrome and toxic epidermal necrolysis in children: a pooled analysis. Pediatrics. 2009;123:297-304.

16. Sontheimer R, Garibaldi R, Gerald G, Krueger M. StevensJohnson syndrome associated with Mycoplasma pneumoniae infections. Arch Dermatol. 1978;114:241-4.

17. Garcia-Doval I, LeCleach L, Bocquet H, Otero X, Roujeau J-C. Toxic epidermal necrolysis and Stevens-Johnson syndrome. Arch Dermatol Am Med Assoc. 2000;136:323-7.

18. Kilmarx PH. Global epidemiology of HIV. Curr Opin I HIV AIDS. 2009;4:240-6.

19. Malaria Control Programme. Epidemiology of malaria in Kenya. Afr J Med Pract. 1994;1:5-6.

20. Mockenhaupt M, Viboud C, Dunant A, Naldi L, Halevy S, Bouwes Bavinck JN, et al. Stevens-Johnson syndrome and toxic epidermal necrolysis: assessment of medication risks with emphasis on recently marketed drugs. The EuroSCAR-study. J Investig Dermatol. The Society for Investigative Dermatology, Inc. 2008;128:35-44.

21. Wafula E, Abinya N, Karanja J, Kaseje D, Musoke R. Clinical guidelines for management and referral of common conditions at levels 4-6 hospitals, vol. III. Nairobi: Ministry of Medical Services and Ministry of Public Health and Sanitation; 2009. 